\title{
Analysis of Interferences in Data Transmission for Wireless Communications Implemented in Vehicular Environments
}

\author{
Valentin Iordache, Razvan Andrei Gheorghiu, Marius Minea \\ Transport Faculty, POLITEHNICA University of Bucharest \\ Bucharest, Romania \\ Email: \{valentin.iordache, marius.minea, andrei.gheorghiu \}@upb.ro
}

\begin{abstract}
This paper aims to provide an image on the usability of low power, short distance standard communications technologies for specific applications, like messaging, in cooperative collision avoidance or emergency vehicles guidance. Specific measurements regarding communications interferences and density have been performed in representative road junctions in Bucharest and the results were used to determine modalities for employing this type of communications for such applications.
\end{abstract}

\section{INTRODUCTION}

$\mathrm{W}$ IRELESS communications are the backbone of many applications in transports, ensuring proper data transfer between different equipment related to traffic management, travel information etc. Moreover, these days critical applications like collision warning systems and route guidance for emergency vehicles use wireless communications to send valuable information onboard selected vehicles. Most part of wireless communications that are being used are those for short \& medium distance, Bluetooth (BT) and Wi-Fi appearing to be the most commonly used. The main issue is they share same frequency bands, which makes the communications interfere with each other. In the literature, many studies regarding this aspect can be found (like [1], [2], [3]), revealing interferences that occur in different scenarios. However, in proper conditions, Bluetooth and Wi-Fi are the cheapest solution for communication and, hence, the first choice.

One convenient feature is the capability of Bluetooth and Wi-Fi devices to send general data, regardless of their connection to an access point (AP) or another device. This data may be used to determine the density of communications in a specific area or on a specific route, and the radio frequency signal power. This information may be then used to determine the feasibility of a communication technique in specific points or areas in the city or outside of it.

This work has been funded by University Politehnica of Bucharest, through the "Excellence Research Grants" Program, UPB - GEX. Identifier: UPB-EXCELENȚ Ă-2016 Research project title "VEHINET - Rețea cu conținut informațional adaptiv la condițiile mediului destinată deplasării inteligente a vehiculelor", Contract number 45/26.09.2016 (acronym: VEHINET).
To search for solutions to a common use of BT and Wi-Fi without critical interferences, a series of measurements have been made in different sites and junctions in Bucharest city, scanning for Wi-Fi communications. The goal was to determine the possibility of implementing another wireless communication technology that would not be affected by these interferences.

\section{WIRELESS COMMUNICATIONS BASICS}

BT and Wi-Fi devices use the same frequencies to communicate. Such links between the transmitter and the receiver of a specific technology might, therefore, be perturbed by another transmission from the other technology. Moreover, the time to transfer a specific length message may, in this case, increase significantly. This is of crucial importance especially for critical messaging in vehicular communication, if a communication point is located on a vehicle traveling with speed s, and the other communication point is either fixed or mobile, then the time the two devices may be in range for communicating is limited. Beside the classic Wi-Fi access points, or communicating devices, there is a set of other electromagnetic devices that may cause interference: microwave ovens, cables associated with satellite receivers, power lines, cordless telephones etc. From the $2.4 \mathrm{GHz}$ and $5 \mathrm{GHz}$ bands, the most crowded with communicating devices is the $2.4 \mathrm{GHz}$ band. In [4], Baccour et al. notes that, from another point of view, interferences might be classified as internal (generated by communicating nodes belonging to the same appliance) or external (generated by sources from exterior). The authors wrote that "The primary outcome of interference is an increase in the packet loss rate, and it is in turn often followed by an increase in the network traffic due to retransmissions, as well as by a decrease in the performance and efficiency of the overall network".

In a context of a low power communicating nodes, external interference may be caused by devices operating in the same frequency range (from other technology), but employing higher transmission powers and thus creating interferences. 
The IEEE 802.15.1 (BT) standard specifies 79 channels, spaced $1 \mathrm{MHz}$, in the range $2402-2480 \mathrm{MHz}$, with center frequency $\mathrm{Fc}=2402+\mathrm{k}$, with $0 \leq \mathrm{k} \leq 78$. Bluetooth uses the Frequency Hopping Spread Spectrum (FHSS) technology to combat interference and fading: it hops 1600 times per second, and therefore it remains at most $625 \mu \mathrm{s}$ in the same channel. Given that only 79 channels are available, on average, one channel is used approximately 20 times each second: this makes interference generated by Bluetooth devices uniformly distributed across the whole $2.4 \mathrm{GHz}$ band.

The Wi-Fi standard uses 2 bands divided into channels: the $2.4 \mathrm{GHz}$ band $(2400-2483.5 \mathrm{MHz})$, for example, is divided into up to 142.2 Crowded Spectrum 27 channels, 2 each of which having a bandwidth of $22 \mathrm{MHz}$. The standard evolved significantly in the last decade (the first version was released in 1997), with data rates increasing from the original $2 \mathrm{Mbit} / \mathrm{s}$ to the $11 \mathrm{Mbit} / \mathrm{s}$ of $802.11 \mathrm{~b}$ (1999), 54 $\mathrm{Mbit} / \mathrm{s}$ of $802.11 \mathrm{~g}$ (2003), up to the $150 \mathrm{Mbit} / \mathrm{s}$ of $802.11 \mathrm{n}$ (2009); and it is still undergoing changes, with the new high-throughput $802.11 \mathrm{ac}$ protocol currently under development. Several works in the literature investigate the impact of IEEE 802.11 communications on the reliability of IEEE 802.15.4 transmissions, and show that wireless sensor networks suffer from high packet loss rates in the presence of Wi-Fi interference.

In [5], Marina Petrova et al. performed a set of measurements to examine the interference of IEEE $802.11 \mathrm{~g} / \mathrm{n}$ on IEEE 802.15 .4 devices. The authors concluded that in an environment with a middle or high IEEE $802.11 \mathrm{n}$ traffic load it is very difficult to guarantee the quality of the nearby operating IEEE 802.15.4 based communicating nodes. Also, even outside of the operating channel the IEEE $802.11 \mathrm{n}$ power is high enough to seriously interfere the IEEE 802.15.4 channels. Some authors also propose techniques to tolerate external interferences [6]. In [4] a taxonomy for external interference mitigation techniques is described (Fig. 1).

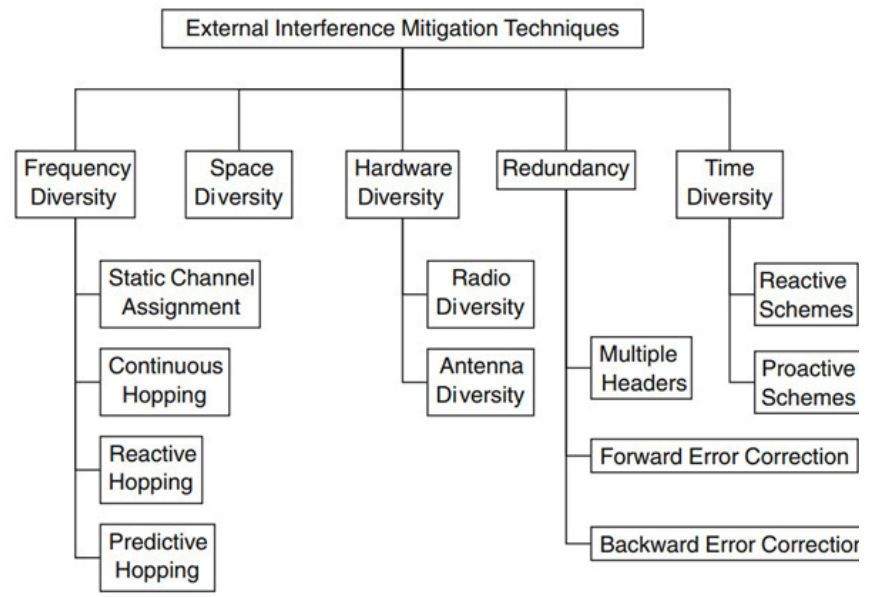

Fig. 1. A taxonomy proposed from literature study for external interference mitigation techniques (source: [4])
Shuaib et al. in [7] show that is necessary to distinguish between uplink and downlink when interference occurs. There are two kinds of devices for IEEE 802.11: access points and terminals. The packets transmission from terminal to access point is defined as "uplink", while the reverse is denoted "downlink". This idea might be useful in traffic information, with systems employing anonymous detection of vehicles, where only "listening" to traffic between passing BT and/or Wi-Fi nodes (vehicles equipped with such technologies) is used to collect information regarding traffic, speed, heading etc. Of course, special filtering and statistics is to be used for obtaining final information regarding traffic. However, in some situations there is no need for very accurate information regarding traffic flow or density (such examples may include: environmental protection techniques of traffic regulation, global information for traffic participants etc.).

Interference may also lead to unpredictable medium access contention times and high latencies, which are also important issues for vehicular communication of critical messages, where guaranteeing high packet delivery rates and limited delay bounds is necessary, and where unreliable connections cannot be tolerated. One reason for this is that vehicles and roadside communicating nodes are not in range for too long. Therefore, the applications in this case should take care of interferences, QoS, and allocate critical messages on less disturbed channels or communication media.

In [8], Hauer, J.H. et al. present the spectrum usage of the above two standards, showing that despite interference mitigation mechanisms like DSSS (Direct Sequence SpreadSpectrum) and "listen-before send" incorporated in both standards, it is well established that their mutual interference can result in notable deterioration of packet delivery performance.

The authors also noticed that in the urban environments transmission failures sometimes span over multiple consecutive 802.15.4 channels, are often correlated in time and substantial losses are typically accompanied by an increase in the noise floor. This suggests that external interference, in particular where there is the omnipresent WLAN and channels are overlapping (Fig. 2), can be a major cause for substantial packet loss in IEEE 802.15.4 vehicular area networks.

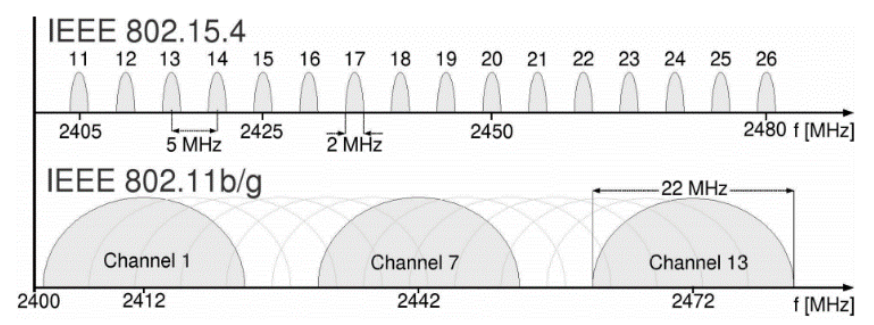

Fig. 2. IEEE 802.15.4 and 802.11 frequencies in the $2.4 \mathrm{GHz}$ ISM band (source: [8]) 
From the above-mentioned tests, it may be concluded that Wi-Fi communications (which may be found in almost every point of an urban road network) produce noise with a negative influence on other wireless communications that might be implemented in the area with the purpose of supporting Intelligent Transport Systems. Therefore, it seems that a major step in introducing a new communication system (regardless of the solution), is to scan the environment for other communications that are already in place and are being impossible to control (e.g. Wi-Fi access points from companies located near the road junctions or bus stops). Such tests may offer an image of the RF environment and provide a proper support for the analysis that will the conclude the best new communication technology that may be used for future applications.

\section{WI-FI COVERAGE - FIELD MEASUREMENTS}

To have some knowledge about the Wi-Fi spectrum coverage that may be found in different junctions in dense urban area, a series of tests have been performed in several junctions in Bucharest city (the largest city in Romania). Highly congested junctions have been chosen near blocks of flats, or company buildings and communications density in these areas has been analysed. As Wi-Fi can send anonymous data that can be identified, Wi-Fi Analyzer application for mobile phones have been chosen, capable of detecting and providing information on Wi-Fi devices in the area. Both frequency bands $(2.4 \mathrm{GHz}$ and $5 \mathrm{GHz}$ ) have been scanned. However, the data obtained for the $5 \mathrm{GHz}$ frequency band is for the moment considered irrelevant, as few communications of this type were detected. The information was obtained as graphs and lists, as presented in Fig. 3 and Fig. 4.

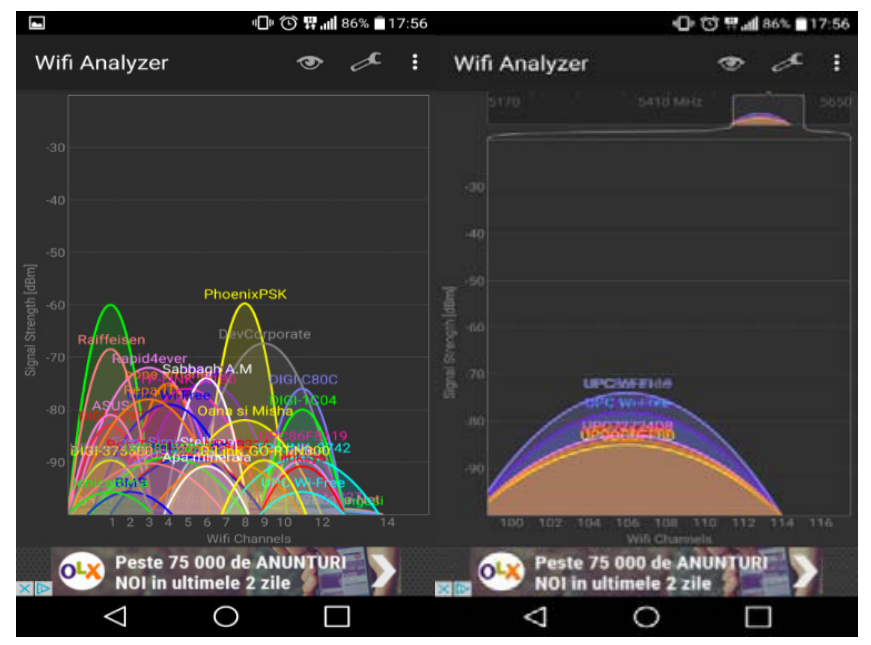

Fig. 3. Example of Wi-Fi data obtained for $2.4 \mathrm{GHz}$ and $5 \mathrm{GHz}$ frequency bands

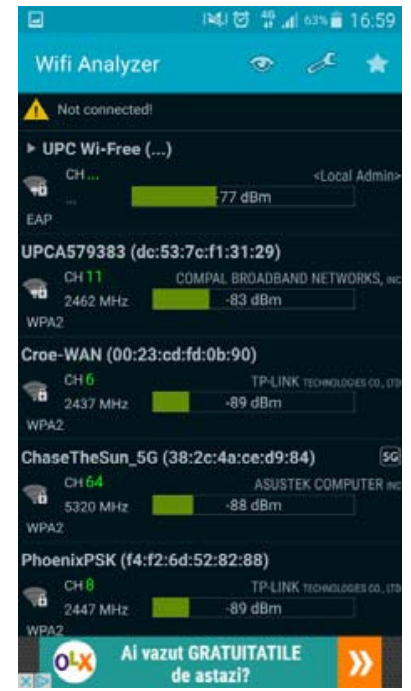

Fig. 4. Example of Wi-Fi data obtained as a detailed list

In the following, the data obtained in three road junctions, in four time intervals will be presented. It is considered relevant to take into account the total number of devices for each Wi-Fi channel, differentiated where possible in 20 $\mathrm{MHz}$ and $40 \mathrm{MHz}$ wide channels, along with the density of Wi-Fi communications on each channel. Afterwards, the maximum signal power for each channel will be presented, as an average for the whole period when measurements took place. The lowest power of -100 was considered for the channels with no communication detected. For convenience, road junctions were named $\mathrm{J} 1-\mathrm{J} 4$.

In Fig. 5 it is noted that the general theory that Wi-Fi channels 1,6 , and 11 are the most used ones [6], [9] is confirmed in real life measurements - this is because they are non-overlapping.

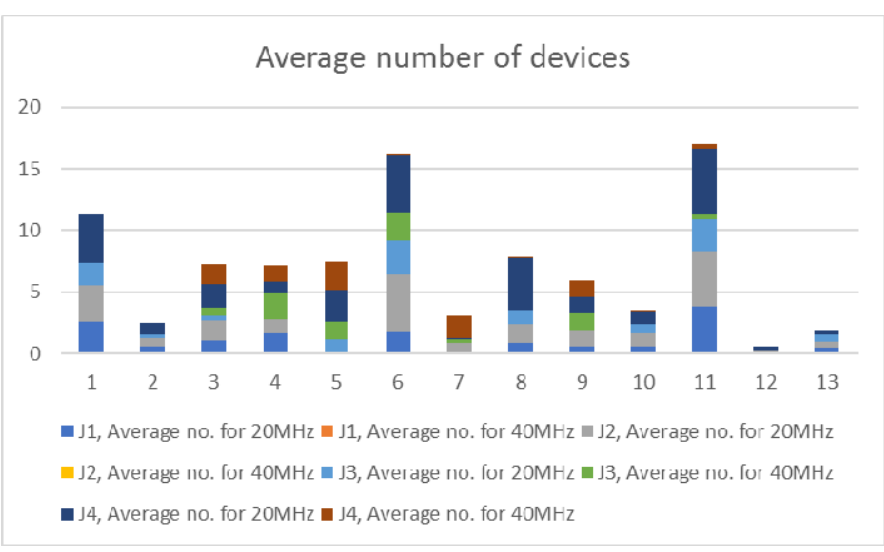

Fig. 5. Average number of devices for each Wi-Fi channel 


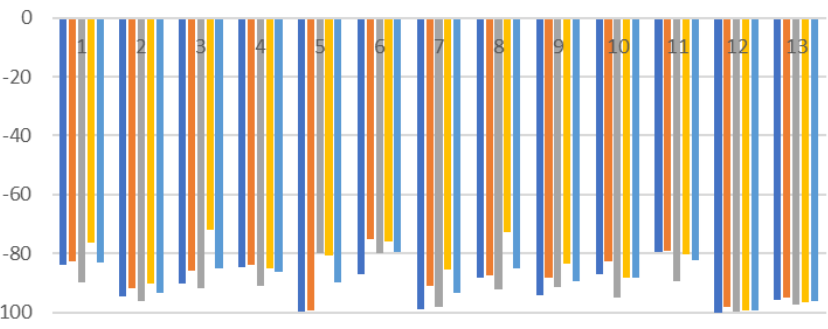

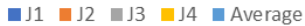

Fig. 6. Average maximum power for each Wi-Fi channel

Fig. 6 presents a comparison for average maximum signal power detected for each channel in each road junction.

Maximum signal power for each junction is provided for each Wi-Fi channel, and the average for all 4 data sets is calculated and represented.

\section{CONCLUSION}

From the tests performed it can be concluded that indeed, Wi-Fi channels 1, 6, and 11 are the most used. This fact has influence mainly on Bluetooth communications, that use three advertising channels overlapping Wi-Fi channels 1 and 6 . Therefore, the implementation of new communications in the proximity of road areas must also consider the existing technologies, besides the application goal, in order to be able to provide a reliable data transfer. Dynamic channel allocation is recommendable for critical applications that do not accept message delaying. Therefore, the involved communication equipment and related protocol should be able to "listen" to all channels before deciding which is best suitable for a specific threshold accepted for the quality of service (message delaying and packet loss). Another solution for specific vehicular applications might be the installation of a roadside unit able to perform these operations (in a RF noisy environment), with the ability to collect information regarding the most crowded frequencies, then to compose a broadcast message recommending the best channels to communicating devices.

In the next period, field measurements will be performed to determine the density of $\mathrm{Wi}-\mathrm{Fi}$ and $\mathrm{BT}$ communications over a determined sector of road (the average number of communicating nodes over a determined distance). Also, another goal is to determine the number of fixed communicating nodes over mobile ones' ratio. This information might be useful in conceiving new communication protocols, aware of the RF environment and more protective regarding message delaying in critical vehicular applications.

Also, it is in the authors' intention to perform Bluetooth and ZigBee data transfer tests in previously tested road junctions, to evaluate and quantify also the influence of $\mathrm{Wi}$ Fi over other wireless data transfer technologies.

\section{REFERENCES}

[1] Freescale Semiconductor, Inc., "Wireless Coexistence in the $2.4 \mathrm{GHz}$ Band", Application Note, Document Number: AN5185, 2015.

[2] S. Silva, S. Soares, T. Fernandes, A. Valente, A. Moreira, "Coexistence and interference tests on a Bluetooth Low Energy frontend", In Proceedings of 2014 Science and Information Conference, SAI 2014, pp. 1014-1018, 2014, DOI: 10.1109/SAI.2014.6918312.

[3] Kaur G., "Bluetooth and Wi-Fi Interference: Simulations and Solutions", International Journal of Advanced Research in Computer and Communication Engineering, Vol. 3, Issue 9, September 2014.

[4] N. Baccour et al., "Radio Link Quality Estimation in Low-Power Wireless Networks", 21 SpringerBriefs in Electrical and Computer Engineering, DOI: 10.1007/978-3-319-00774-8_2, 2013

[5] M. Petrova, L. Wu, P. Mähönen, J. Riihijärvi, "Interference measurements on performance degradation between colocated IEEE $802.11 \mathrm{~g} / \mathrm{n}$ and ieee 802.15.4 networks". In: Proceedings of the international conference on networking (ICN), pp 93-98, 2007

[6] Yang D, Xu Y, Gidlund M, "Wireless coexistence between IEEE 802.11- and IEEE 802.15.4-based networks: a survey". Int J Distrib Sens Netw (IJDSN) 2011:17pp, 2011

[7] Shuaib K., Boulmalf M., Sallabi F., and Lakas A., "Co-existence of Zigbee and WLAN, a performance study," in Proceedings of the International Conference on Wireless and Optical Communications Networks (IFIP '06), Bangalore, India, April 2006.

[8] Hauer JH, Handziski V, Wolisz A, "Experimental study of the impact of WLAN interference on IEEE 802.15 .4 body area networks". In: Proceedings of the $6^{\text {th }}$ European conference on wireless sensor networks (EWSN), pp 17-32, 2009

[9] Liang CJM, Priyantha NB, Liu J, Terzis A, "Surviving Wi-Fi interference in low power zigbee networks". In: Proceedings of the $8^{\text {th }}$ ACM conference on embedded networked sensor systems (SenSys '10). ACM, New York, pp 309-322, 2010

[10] Chen H, Cui L, Lu S, "An experimental study of the multiple channels and channel switching in wireless sensor networks". In: Proceedings of the $4^{\text {th }}$ international symposium on innovations and real-time applications of distributed sensor networks (IRADSN), pp 54-61. 2009

[11] Nethi S, Nieminen J, Jäntti R, "Exploitation of multi-channel communications in industrial wireless sensor applications: avoiding interference and enabling coexistence". In: Proceedings of the IEEE wireless communications and networking conference (WCNC), pp 345-350, 2011

[12] Mangir, T., Sarakbi, L., Younan, H., "Analyzing the impact on Wi-Fi interference on ZigBee networks based on real-time experiments." International Journal of distributed and parallel networks, Vol.2, No.4, July 2011 